\title{
Influencia de las Mezclas de gases Ar-He y Ar-He-O en la Soldabilidad de la Aleación de Aluminio AA5083-O usando Proceso GMAW-P Automatizado
}

\author{
(Influence of Gas Mixtures Ar-He and Ar-He-O on weldability of aluminum alloy AA5083- O using Automated GMAW-P)
}

\author{
Enrique Esteban Niebles Nuñez ${ }^{1}$, Jimy Unfried Silgado ${ }^{1}$, Jaime Elías Torres Salcedo ${ }^{2}$, Antonio J. Ramírez ${ }^{3}$ \\ ${ }^{1}$ Universidad Autónoma del Caribe, Programa de Ingeniería Mecánica, Barranquilla, Colombia.eniebles@uac.edu.co, jimy. \\ unfried@uac.edu.co \\ ${ }^{2}$ Universidad del Norte, Programa de Doctorado en Ingeniería Mecánica, Barranquilla, Colombia.jtorres@uninorte.edu.co \\ ${ }^{3}$ Metals characterization and processing laboratory, Brazilian Nanotechnology National Laboratory, CNPEM/ABTLuS. Campinas, \\ Brasil.
}

\section{Resumen}

La problemática en las empresas fabricantes de estructuras y construcciones soldadas en aluminio se centra en la disminución de las propiedades mecánicas de las uniones soldadas influenciada por el aporte térmico en las regiones de soldadura; así como, en los efectos de las discontinuidades y defectos, los altos costos de reparación de soldaduras y la baja productividad. El objetivo de este trabajo es evaluar la influencia de las mezclas de gases 80Ar20He (MG1), 60Ar40He (MG2), 80Ar19He1O (MG3), 60Ar39He1O (MG4), sobre la soldabilidad de la aleación de aluminio AA5083-O utilizando un material de aporte ER5183 y el proceso GMAW-P automatizado. La metodología incluyó una fase de parametrización de soldaduras, la caracterización del metal base y las regiones de soldadura. El análisis de resultados se llevó a cabo en términos de la evolución microestructural en las regiones de soldadura y evaluación de las propiedades como dureza, resistencia y elongación, así como la evaluación de discontinuidades y defectos de la unión, costos y velocidad de soldadura. Los resultados muestran que las mezclas de gas que contienen $\mathrm{O}_{2}$ favorecen significativamente la soldabilidad de la aleación en estudio.

Palabras clave: Soldabilidad y Estabilidad; Gas de protección; Aleaciones de aluminio naval; Microestructura de soldadura; Propiedades mecánicas; Integridad de soldadura.

\begin{abstract}
Problems of industries and manufacturers of welded structures in aluminum alloys are centered on reduction of mechanical properties in welded joints due to heat input effects on heat affected zone (HAZ); as well as, effects of discontinuities and metallurgical defects, high cost repairs, and low productivity. The aim of this work is to evaluate the influence of gas mixtures 80Ar20He (MG1), 60Ar40He (MG2), 80Ar19HelO $(M G 3)$, and 60Ar39He1O (MG4), on aluminum alloy AA5083-O weldability using filler metal ER5183 automated GMAW-P process. Methodology has included a factorial experiment design, customization of welding parameters, characterization of base metal and welding regions. Analysis of results were carried out based on microstructure evaluation of welding regions, measurements of hardness and tensile properties, observations of discontinuities and defects, costs evaluation, and welding speed. Results show that gas mixtures with $\mathrm{O}_{2}$ are highly beneficial to obtain good weldability of studied alloy.
\end{abstract}

Key-words: Weldability and stability; aluminum alloys for naval applications; Welded microstructure; mechanical properties; Integrity welding.

\section{Introducción}

Los gases de protección utilizados en la soldadura de aleaciones de aluminio con proceso de soldadura de arco protegido con gas y electrodo consumible - GMAW son el argón (Ar), helio (He), mezclas binarias de Ar-He en variadas proporciones y mezclas ternarias de $\mathrm{Ar}-\mathrm{He}-\mathrm{O}_{2}$ en variadas proporciones. Al soldar con argón se tiene un cordón más ancho

Recebido em 27/05/2013, texto final em 05/09/2014.

DOI: http://dx.doi.org/10.1590/0104-9224/SI1903.06 en la superficie y menos profundo que con helio; se tiene un arco más estable y fácil de iniciar por su bajo potencial de ionización (15,5 eV para el argón y 24,5eV para el helio) y se ejerce una acción limpiadora de óxidos en la superficie [1-3]. La soldadura con argón presenta mayor tendencia a la formación de poros y susceptibilidad al agrietamiento en caliente. La soldadura con helio en comparación con el argón permite mayor velocidad de soldadura, penetración más profunda, produce más salpicaduras y requiere una limpieza de la tobera más frecuente $[2,4]$. Además el helio por su baja densidad requiere mayor flujo de gas para mantener la eficacia de la protección y a medida que aumenta el contenido de helio se disminuye la formación de poros presentes en el depósito de soldadura, debido a la mayor 
Tabla 1. Propiedades mecánicas de aleaciones de aluminio de aplicación naval AA5083-0. Láminas y platinas [12-15].

\begin{tabular}{|c|c|c|c|c|c|}
\hline Aleación AA & Espesor [mm] & $\begin{array}{c}\text { Resistencia a fluencia } \\
\mathbf{S}_{\mathbf{v}}[\mathbf{M P a}]\end{array}$ & $\begin{array}{c}\text { Resistencia última } \\
\mathbf{S}_{\mathbf{u}}[\mathbf{M P a}]\end{array}$ & $\begin{array}{c}\text { Elongación en } \\
\mathbf{5 0 m m}[\mathbf{\%}]\end{array}$ & $\begin{array}{c}\text { Dureza Vickers } \\
{[\mathbf{H V}]}\end{array}$ \\
\hline $\mathbf{5 0 8 3 - O}$ & $1,3-38,1$ & $125-200$ & $275-350$ & 16 & 87 \\
$1,2-6,3$ & 125 & 275 & -- & -- \\
\hline $\begin{array}{c}\mathbf{5 0 8 3 - H 1 1 6 , ~ H 3 2 1 ,} \\
\text { O Soldada }\end{array}$ & 6,3 a 38 & 115 & 270 & - & \\
\hline
\end{tabular}

fluidez del baño de soldadura que facilita la desgasificación de hidrógeno [3-6]. La mezcla de gases Ar-He favorecen por efecto del helio el ancho del cordón, la penetración de soldadura y mayor depósito por área al tener mayor aporte térmico permitiendo el uso de alambres de mayor diámetro durante la soldadura [1,2]. Contenidos de oxígeno $\left(\mathrm{O}_{2}\right)$ en porcentajes mayores al $2 \%$ afectan negativamente la soldadura, debido a que el oxígeno en el baño de fusión se combina con átomos de aluminio generando inclusiones solidas de $\mathrm{Al}_{2} \mathrm{O}_{3}$, reduciendo las propiedades mecánicas de la unión soldada $[5,7]$. Fortain et al. [5], a través de desarrollos experimentales en soldaduras de aluminio con proceso GMAW-P bajo mezclas con contenido de $\mathrm{O}_{2}$ en proporciones de $0,01 \%$ al $1,8 \%$, He en proporciones de $20 \%$ a $98,2 \%$ y balance argón muestran como la adición de $\mathrm{O}_{2}$ a diferentes mezclas de Ar-He favorece la productividad en términos de velocidad y penetración de soldadura, además con la adición de $\mathrm{O}_{2}$ se disminuye ligeramente la resistencia a la tracción.

En la soldadura de aleaciones de aluminio es posible hoy día encontrar diversidad de equipos que permiten ajustar los parámetros de soldadura y sus curvas características con GMAW. $\mathrm{Al}$ soldar en el modo de transferencia spray, la transferencia es por diminutas gotas del mismo tamaño transferidas a través del arco desde menos de un ciento hasta varios cientos de veces por segundo, gracias a la densidad de alta corriente y altos voltajes que produce un calor intenso con altas tasas de deposición, favoreciendo altos indicadores de productividad $[1,5]$. En las soldaduras de aluminio con GMAW transferencia pulsada es posible trabajar con equipos sinérgicos, en los cuales para cada combinación de metal base y aporte, espesor y gas se ajustan automáticamente los parámetros en función de la frecuencia, extensión libre del alambre, voltaje y longitud de arco, velocidad de alimentación de alambre y corriente de soldadura [2]. La transferencia por arco pulsado se da en intervalos espaciados con el amperaje pulsante entre altos picos preajustados y bajos niveles de fondo. La fuente de poder provee dos niveles de corriente, un amperaje pico se ajusta sobre el punto de transferencia por spray con una intensidad mayor a la corriente de transición y el amperaje de fondo se ajusta en el intervalo de la transferencia globular, la combinación de las dos corrientes produce un arco estable con una transferencia de metal controlada similar a la transferencia spray. Esta permite el uso de alambres de mayor diámetro y avance más uniforme. Además, con la transferencia pulsada se tiene la posibilidad de soldar con corrientes medias bajas sin incurrir en la transferencia en cortocircuito, lo cual es muy útil en soldaduras de embarcaciones navales donde comúnmente se suelda fuera de posición plana. La soldadura en modo pulsado es recomendada cuando se desea altos estándares de calidad, mejorando las propiedades mecánicas de la unión soldada, aun cuando reduce los indicadores de productividad por la disminución de las velocidades de soldadura $[1,5,8]$. En la soldadura de aluminios el uso de respaldos cerámicos contribuye a reducir la contaminación gaseosa, brinda mejor apariencia del cordón de respaldo, tienen resistencia al choque térmico y sin absorber humedad, dando mayor velocidad de soldadura y eficiencia en el tiempo de producción, reduciendo costos de producción [9-11].

Las aleaciones AA5083 bajo la condición O, soldadas con proceso GMAW convencional y gas argón, no reducen su resistencia, véase la Tabla 1. Esto es, los indicadores de resistencia como condición O-soldada son muy similares a las propiedades de resistencia en la condición O-no soldado.

Tomando como base lo anteriormente descrito, este artículo tiene como objetivo seleccionar cuál mezcla de Ar-He o Ar$\mathrm{He}-\mathrm{O}_{2}$ bajo diferentes aportes térmicos, influye de manera positiva sobre la soldabilidad de la aleación de aluminio AA5083-O con proceso GMAW-P automatizado. La selección se realiza en términos de la determinación de propiedades como dureza, resistencia y elongación; observación de presencia de poros $y$ análisis microestructural con microscopía óptica en las regiones de soldadura.

\section{Materiales y Métodos}

\subsection{Materiales e insumos}

En este trabajo se usó como material de base la aleación AA5083-O y material de aporte AlMg4.5Mn con clasificación AWS A5.10:99 ER5183 y diámetro Ø1,2mm. Los gases de protección MG1:80Ar20He, MG2:60Ar40He, MG3:80Ar19He1O,$\quad$ MG4:60Ar39He1O $\mathrm{H}_{2}$ utilizados en el estudio no son comerciales, por lo cual fueron fabricados y suministrados por una reconocida empresa multinacional.

\subsection{Composición química, propiedades mecánicas $\mathbf{y}$ caracterización microestructural del metal base}

La caracterización de la composición química, microestructural y propiedades mecánicas de la aleación en estudio se muestran en la Tabla 2. La caracterización microestructural en términos de la forma y orientación de los granos se obtuvo mediante análisis de imágenes obtenidas con microscopia óptica en cada orientación de laminado con un microscopio óptico marca Olympus ${ }^{\circledR}$ modelo G50X a 200X. Ataque Keller's. Los especímenes de prueba para ensayos de tensión del metal base fueron extraídos paralelos a la orientación 
Tabla 2. Caracterización de la composición química, microestructural y propiedades mecánicas del metal base aleación AA5083-O. MO=Microscopia Óptica

Composición química metal base AA 5083-O. \% en peso.

Valores obtenidos por espectrometría de emisión óptica en los laboratorios de la Universidad de Antioquia

\begin{tabular}{|c|c|c|c|c|c|c|c|c|c|c|c|}
\hline Aleación & Si & $\mathbf{F e}$ & $\mathrm{Cu}$ & Mn & Mg & $\mathrm{Cr}$ & Zn & $\mathbf{T i}$ & \multicolumn{2}{|c|}{ Otros elementos } & Al \\
\hline $5083-\mathrm{O}$ & 0,088 & 0,253 & 0,008 & 0,737 & 4,436 & 0,059 & 0,109 & 0,01 & $\begin{array}{c}\mathrm{Ni}=0,005 \\
\mathrm{~V}=0,008\end{array}$ & $\begin{array}{l}\mathrm{Ca}=0,002 \\
\mathrm{Zr}=0,002\end{array}$ & 94,281 \\
\hline \multicolumn{4}{|c|}{ Imagen MO de la microestructura } & \multicolumn{8}{|c|}{ Resultados de Propiedades Mecánicas metal base } \\
\hline$e^{\circ}$ & & & & & \multicolumn{3}{|c|}{$\begin{array}{c}\text { Resistencia a } \\
\text { fluencia } \overline{\mathrm{Sy}}[\mathrm{Mpa}]\end{array}$} & \multicolumn{2}{|c|}{$\begin{array}{c}\text { Resistencia } \\
\text { última } \overline{\mathrm{Su}}[\mathrm{MPa}]\end{array}$} & $\begin{array}{c}\text { Alargamiento } \\
\overline{\text { E2in }(\%)}\end{array}$ & $\frac{\text { Dureza Vickers }}{\text { MHV }_{\mathbf{1 0 0 g} / \mathbf{1 0 s}}}$ \\
\hline & & & & & \multicolumn{3}{|c|}{178,2} & \multicolumn{2}{|c|}{331,8} & 16,8 & \multirow[t]{2}{*}{85,8} \\
\hline$\therefore \mathrm{MBE}_{2} 200 \mathrm{X}$ & & & $\overline{50 \mu \mathrm{m}}$ & & & & & & & & \\
\hline
\end{tabular}

de laminado y realizadas las pruebas en una máquina universal de ensayos marca UH-100A, Shimadzu ${ }^{\circledR}$, con una velocidad de cabezal de $4 \mathrm{~mm} / \mathrm{min}$. Los valores de microdureza Vickers fueron determinados usando una carga de $100 \mathrm{~g}$ durante $10 \mathrm{~s}$, las cuales se midieron a lo largo de la orientación longitudinal de laminado con un microdurómetro Vickers Akashi ${ }^{\circledR}$ modelo MVK-HO.

El análisis de los resultados mostrados en las tabla 2, permite afirmar que la identidad de la aleación utilizada para el desarrollo de la investigación es coincidente con la aleación AA5083-O con una resistencia promedio a fluencia de 178,2 $\mathrm{MPa}$, resistencia promedio última de 331,8 $\mathrm{MPa}$, ductilidad promedio en secciones de deformación de 2,0 pulgadas del $16,8 \%$ y microdureza Vickers promedio de 85,8 ; observándose además una estructura de grano en estado recocido para este tipo de aleación $[15,16]$.

\subsection{Parámetros del proceso de soldadura y cálculo del aporte térmico para las juntas soldadas}

El diseño de la junta y la secuencia de llenado de cordones de soldadura se muestran en la Figura 1. Las soldaduras son realizadas con GMAW-P automatizado, en posición plana (1G) bajo las mismas condiciones de soldeo. Los cordones de soldaduras fueron ejecutados sin oscilación de la pistola. Para la parametrización de soldadura es necesario variar y ajustar los parámetros de soldadura que configuren los diferentes aportes térmicos (HI) para cada mezcla de gas, en la cual es operativamente soldable la aleación, manteniendo a la vez una diferencia del $10 \%$ o mayor entre aportes térmicos por mezcla de gas y así dar validez a la comparación de resultados en el estudio. Esto es, definida la habilidad y experiencia del soldador se tuvo en cuenta: estabilidad de arco, llenado de soldadura, excesos de salpicadura, calentamiento excesivo de la tobera, falta o exceso de penetración, contorno de soldadura.
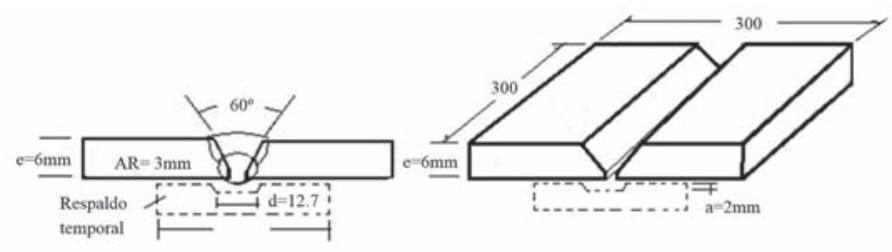

Figura 1.Diseño de junta y secuencia de cordones para aplicación de soldaduras.

La fuente de poder utilizada en el desarrollo de soldaduras corresponde con un equipo de soldadura XMT® $350 \mathrm{CC} / \mathrm{CV}$, con alimentador 22A y mando de control Óptima ${ }^{\circledR}$ para los parámetros de soldadura GMAW-P. Con el control Optima ${ }^{\circledR}$ en el modo manual de GMAW-P es posible ajustar y fijar los siguientes parámetros de soldadura: Corriente de inicio $\mathrm{I}_{\mathrm{i}}$, corriente pico $I_{p}$, corriente base $I_{b}$, tiempo pico de pulso $t_{p} y$ frecuencia de pulso $f_{p}$. Como sistema automático para el control de movimientos y velocidades de soldadura se utilizó el sistema Railtrac ${ }^{\circledR}$. Los parámetros de soldadura pueden, así mismo, ser obtenidos mediante procesamiento de señales eléctricas apoyados en sistemas de adquisición de datos. Para determinar el valor de la corriente media, se debe tener en cuenta el tipo de onda suministrada por el equipo al realizar las soldaduras, pudiendo ser de tipo rectangular o trapezoidal [17-18]. Como no fue del alcance del trabajo identificar el tipo de señal de onda utilizada y medir los parámetros de soldadura mediante procesamiento de señales eléctricas en sistema de adquisición de datos, estos fueron medidos apoyados en la herramienta control Optima ${ }^{\circledR}$, asumiendo una señal de onda rectangular [17-20].

La frecuencia de pulso $\left(f_{p}\right)$ se determinó mediante la Ecuación 1, se percibe que al incrementar la frecuencia aumenta la longitud de arco, la corriente media $\mathrm{I}_{\mathrm{m}}$, $\mathrm{y}$ el calor suministrado [18-20]. 
$f_{p}=\frac{1}{\left(t_{b}+t_{p}\right)}$

El valor de la corriente media $I_{m}$ para señales de ondas perfectamente rectangulares es calculado mediante la Ecuación 2 [18,21-23] e involucra parámetros de soldadura como: corriente base $I_{b}$, corriente pico $I_{p}$, tiempo pico de pulso $t_{p}$, tiempo base de pulso $t_{\mathrm{p}}$ y frecuencia de pulso $f_{p}$.

$I_{m}=\frac{I_{b} t_{b}+I_{p} t_{p}}{t_{b}+t_{p}}$

Un aumento del valor de corriente pico incrementa la corriente media y la penetración de soldadura $[19,20]$. La corriente base tiene influencia en la temperatura de transferencia del metal precalentando el alambre, definiendo la fluidez del charco de soldadura, el tamaño de la gota, ancho del baño fundido y humectación en el cordón de soldadura $[17,18]$. Cuando se incrementa la corriente base $\mathrm{I}_{\mathrm{b}}$ se incrementa la longitud de arco, la corriente media $\mathrm{I}_{\mathrm{m}}$, la fluidez del charco de soldadura, penetración y calor suministrado, además se disminuye la extensión libre del electrodo $[19,20]$. Cuando se incrementa el tiempo pico se incrementa la longitud de arco, la corriente media $I_{m}$, el ancho de cono del arco, y el calor suministrado. Cuando se incrementa el tiempo base se disminuye la longitud de arco, la corriente media $\mathrm{I}_{\mathrm{m}}$, el ancho de cono del arco y el calor suministrado, además se incrementa la extensión del electrodo $[19,20]$.

La eficiencia de proceso [23] se calcula en función del factor hipotético mediante la Ecuación 3:

$\eta_{\mathrm{a}}=94.52-0.118 \mathrm{I}_{\mathrm{m}}-107.61 \phi+0.348 \mathrm{I}_{\mathrm{m}} \phi$

El factor adimensional se basa en el análisis del balance de energía del proceso GMAW-P y combina parámetros del pulso $\mathrm{I}_{\mathrm{p}}, \mathrm{I}_{\mathrm{b}}, f_{p} \mathrm{y} \mathrm{t}_{\mathrm{b}}$. El factor es utilizado en el análisis $\mathrm{y}$ control de la estabilidad del arco mediante relaciones empíricas que correlacionan, además, la corriente media $I_{m}$ y el voltaje, permitiendo determinar así características de arco como son: la longitud de arco, diámetro de la raíz y base del arco las cuales tienen influencia sobre la operatividad y calidad de soldadura [23-26]. Este factor es calculado acorde con la Ecuación 4.

$\phi=\left[\left(\frac{\mathrm{I}_{\mathrm{b}}}{\mathrm{I}_{\mathrm{p}}}\right) \cdot f_{p} \cdot \mathrm{t}_{\mathrm{b}}\right]$

El aporte térmico teórico [21, 23, 27] que recibe el metal base es calculado, a partir de la potencia media aritmética [28], la eficiencia del proceso y la velocidad de soldadura, acorde con la Ecuación 5:

Aporte térmico $(\mathrm{HI})=\frac{\eta_{a} \mathrm{I}_{\mathrm{m}} \mathrm{V}_{\mathrm{m}} * 60}{\mathrm{~V}_{\mathrm{s}} * 1000} \quad[\mathrm{~kJ} / \mathrm{cm}]$

Dónde: $\eta a$ es la eficiencia del proceso de soldadura, $\mathrm{I}_{\mathrm{m}}$ es la Intensidad media de corriente en amperios, $\mathrm{V}_{\mathrm{m}}$ es la tensión media de arco en Voltios $\mathrm{y}_{\mathrm{s}}$ es la velocidad de avance de la antorcha en $\mathrm{cm} / \mathrm{min}$.

\subsection{Caracterización mecánica y microestructural de la unión soldada}

Una vez ejecutadas las soldaduras se verifica el cumplimiento de los criterios de aceptación de los cupones soldados por medio de ensayos no destructivos-END tales como, inspección visual y rayos-X. Para la localización de especímenes de tensión y análisis microestructural, así como el corte de los mismos, se toma como base lo especificado en la norma AWS D1.2 [29] y las necesidades de investigación. Los especímenes de prueba para ensayos de tensión en las juntas soldadas fueron extraídos transversales al cordón de soldadura y realizadas las pruebas en una maquina universal de ensayos marca UH-100A, Shimadzu ${ }^{\circledR}$, con una velocidad de cabezal en la máquina ensayo universal de $4 \mathrm{~mm} / \mathrm{min}$. Los valores de microdureza de las regiones de soldadura fueron medidos transversales al cordón de soldadura y a $2 \mathrm{~mm}$ de la superficie de la cara de soldadura y $1,5 \mathrm{~mm}$ de la superficie de la raíz de la soldadura para las juntas soldadas, como se muestran en la figura 2. El barrido de microdurezas de las soldaduras se realizó aplicando una carga de 100gr durante 10 s con un paso de $0,15 \mathrm{~mm}$, valor de paso mayor a 2,5 veces el promedio del tamaño de la diagonal de huella, acorde con lo establecido en la norma ASTM-E384 [30]. La extensión de cada región de soldadura es determinada mediante la lectura de las durezas, tendencia de los valores, la medida del número de indentaciones por el paso y observación con microscopia óptica.

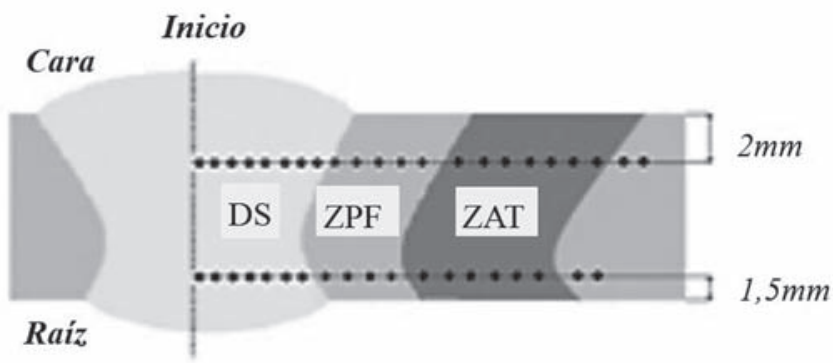

Figura 2. Esquema del barrido de microdureza MHV para juntas soldadas. $\mathrm{DS}=$ Deposito de soldadura, $\mathrm{ZPF}=\mathrm{Zona}$ parcialmente fundida, $\mathrm{ZAT}=$ Zona Afectada térmicamente

Macrografías fueron obtenidas con estereoscopio marca Olympus ${ }^{\circledR}$ modelo Z60. Se utilizó como reactivo para macroataque de la junta el reactivo Tucker, aplicado durante 15 segundos. Para revelar la estructura general de las muestras se utiliza el reactivo Keller's durante 60 segundos [16,31].

\section{Resultados y Discusión}

La parametrización de soldadura bajo diferentes mezclas de gas condujo a los resultados mostrados en la Tabla 3. Aun cuando las probetas fueron sometidas a iguales condiciones de restricciones mecánicas durante el desarrollo de las soldaduras, bajo la protección de las diferentes mezclas de gases, se presentaron distorsiones menores en los cupones de prueba (unas un poco mayor que las otras) por efectos térmicos que ocasionaron variaciones de longitud de arco. Estas variaciones 
confluyeron en variaciones de los valores de voltaje obtenidos para cada aporte térmico.

Para el análisis microestructural y de propiedades mecánicas es importante tener en cuenta la diferencia relativa porcentual presente entre los promedios de los aportes térmicos alto HIa y bajo HIb, para cada mezcla de gas en estudio, que garanticen posibles diferencias en la comparación de resultados obtenidos. Al comparar los valores de los aportes térmicos HIa y HIb, obtenidos en cada una de las soldaduras para cada mezcla de gas, se encuentran diferencias relativas porcentuales: del $24,2 \%$ bajo la mezcla MG3, del $9.5 \%$ y $10,8 \%$ bajo las mezclas MG1 y MG4 y del 4,5\% bajo la mezcla MG2. No fue posible obtener diferencias mayores entre los aportes térmicos alto y bajo para las soldaduras bajo la protección MG2; dado que al modificar los parámetros de soldadura para obtener una diferencia alrededor del $10 \%$, no era operativamente soldable la junta en este estudio.

De la Tabla 3 se puede observar además, que los mayores valores de aporte térmico en los cuales fue operativamente soldable la junta, se obtuvieron con las mezclas que contienen oxígeno, en especial con la mezcla MG3: $80 \mathrm{Ar} 19 \mathrm{He}_{2} \mathrm{O}_{2}$ con un aporte térmico . Los menores aportes térmicos en los cuales fue operativamente soldable la junta, se obtuvieron con la mezcla MG1:80Ar20He con un aporte térmico y un aporte térmico . Se presentan diferencias mayores del $10 \%$ entre los valores de velocidad de soldadura para los diferentes aportes térmicos bajo las diferentes mezclas de gases, a excepción de la mezcla MG1. La mayor velocidad de soldadura en promedio se obtuvo con la mezcla MG3.

\section{1.: Caracterización mecánica de las uniones soldadas}

Del análisis de la curva de esfuerzo vs. deformación a partir del ensayo de resistencia a la tracción se obtuvieron los indicadores de la resistencia a fluencia (Sy), resistencia última $(\mathrm{Su})$ y variación de la ductilidad en secciones de deformación $(\varepsilon)$ en una $(1,0)$ y dos $(2,0)$ pulgadas. Se calculó además la eficiencia $(\eta)$ de la junta definida como la relación entre el valor de resistencia de la unión soldada y el valor de resistencia del metal

Tabla 3. Parámetros experimentales de soldaduras. Valores promedios. HIa $=$ Aporte térmico alto, $\mathrm{HIb}=\mathrm{Aporte}$ térmico bajo, $\eta_{\mathrm{a}}=$ eficiencia del proceso, $\mathrm{t}_{\mathrm{p}}=$ Tiempo pico de pulso, $\mathrm{I}_{\mathrm{p}}=$ Corriente pico, $f_{\mathrm{p}}=$ Frecuencia de pulso, $\mathrm{V}=$ Voltaje, $\mathrm{I}_{\mathrm{m}}=$ Corriente media, $v_{\mathrm{a}}$ $=$ Velocidad de alimentación de alambre, $v_{s}=$ Velocidad de soldadura.

\begin{tabular}{|c|c|c|c|c|c|c|c|c|c|}
\hline Mezcla de gas & $\overline{\boldsymbol{H I}}[\mathrm{kJ} / \mathrm{cm}]$ & $\overline{\eta_{a}}$ & $\overline{t_{p}}[\mathrm{~ms}]$ & $\overline{I_{p}}[\mathrm{~A}]$ & $\overline{f_{p}}[\mathrm{~Hz}]$ & $\bar{V}[\mathrm{~V}]$ & $\overline{I_{m}}[\mathrm{~A}]$ & $\overline{v_{a}}[\mathrm{~m} / \mathrm{min}]$ & $\bar{v}_{s}[\mathrm{~cm} / \mathrm{min}]$ \\
\hline \multirow{2}{*}{ MG1: $80 \mathrm{Ar} 20 \mathrm{He}$} & $H I a=2,42 \pm 0,13$ & 0,61 & 1,5 & 250 & 240 & 19,8 & 160,4 & 5,5 & 48 \\
\hline & $H I b=2,19 \pm 0,04$ & 0,60 & 1,6 & 246 & 134 & 20,8 & 129,7 & 4,5 & 44 \\
\hline \multirow{2}{*}{ MG2: $60 \mathrm{Ar} 40 \mathrm{He}$} & $H I a=2,91 \pm 0,02$ & 0,60 & 1,6 & 246 & 134 & 21,9 & 129,7 & 4,5 & 35 \\
\hline & $H I b=2,78 \pm 0,13$ & 0,62 & 2,2 & 250 & 180 & 22,3 & 165,4 & 5,5 & 49 \\
\hline \multirow{2}{*}{ MG3: $80 \mathrm{Ar} 19 \mathrm{HelO}_{2}$} & $H I a=3,18 \pm 0,07$ & 0,65 & 2,4 & 248 & 246 & 24,9 & 187,4 & 5,5 & 57 \\
\hline & $H I b=2,41 \pm 0,15$ & 0,61 & 2,0 & 240 & 140 & 22,0 & 137,3 & 4,5 & 46 \\
\hline \multirow{2}{*}{ MG4: $60 \mathrm{Ar} 39 \mathrm{He} \mathrm{O}_{2}$} & $H I a=3,14 \pm 0,02$ & 0,63 & 2,0 & 250 & 240 & 23,4 & 177,2 & 5,5 & 50 \\
\hline & $H I b=2,80 \pm 0,04$ & 0,60 & 1,7 & 246 & 134 & 22,6 & 131,3 & 4,5 & 38 \\
\hline
\end{tabular}

Tabla 4. Resultados de la evaluación de discontinuidades para las soldaduras experimentales.

\begin{tabular}{|c|c|c|c|}
\hline $\begin{array}{l}\text { Mezcla } \\
\text { de gas }\end{array}$ & $\overline{H I}[\mathrm{~kJ} / \mathrm{cm}]$ & $\begin{array}{l}\text { Resultados rayos-X en } \\
\text { región tensionada }\end{array}$ & Inspección visual zona fractura \\
\hline MG1: & $H I a=2,42 \pm 0,13$ & Porosidad & Poros pequeños y aislados Porosidad \\
\hline $80 \mathrm{Ar} 20 \mathrm{He}$ & $H I b=2,19 \pm 0,04$ & Porosidad excesiva & excesiva \\
\hline $\begin{array}{l}\text { MG2: } \\
\text { 60Ar40He }\end{array}$ & $\begin{array}{l}H I a=2,91 \pm 0,02 \\
H I b=2,78 \pm 0,13\end{array}$ & $\begin{array}{l}\text { Porosidad excesiva } \\
\text { Porosidad }\end{array}$ & $\begin{array}{l}\text { Porosidad excesiva } \\
\text { Poros pequeños }\end{array}$ \\
\hline MG3: $80 \mathrm{Ar} 19 \mathrm{HelO}_{2}$ & $\begin{array}{l}H I a=3,18 \pm 0,07 \\
H I b=2,41 \pm 0,15\end{array}$ & $\begin{array}{l}\text { No se observan poros } \\
\text { Porosidad }\end{array}$ & $\begin{array}{c}\text { No se observan poros } \\
\text { Porosidad aislada }\end{array}$ \\
\hline MG4: $60 \mathrm{Ar} 39 \mathrm{He} \mathrm{O}_{2}$ & $\begin{array}{l}H I a=3,14 \pm 0,02 \\
H I b=2,80 \pm 0,04\end{array}$ & No se observan poros & $\begin{array}{c}\text { Se observan partículas no identificadas con } \\
\text { Microscopia Óptica. }\end{array}$ \\
\hline
\end{tabular}

Tabla 5. Indicadores de resistencia mecánica de juntas soldadas de AA5083-O.

\begin{tabular}{|c|c|c|c|c|c|c|c|c|c|}
\hline Mezcla de gas & $\begin{array}{c}\overline{\boldsymbol{H I}} \\
{[\mathrm{kJ} / \mathrm{cm}]}\end{array}$ & $\begin{array}{c}\overline{\boldsymbol{S y}} \\
(\mathrm{MPa})\end{array}$ & $\begin{array}{l}\bar{\eta}_{s_{y}} \\
{[\%]}\end{array}$ & $\begin{array}{c}\overline{\boldsymbol{S u}} \\
(\mathrm{MPa})\end{array}$ & $\begin{array}{l}\bar{\eta}_{s_{u}} \\
{[\%]}\end{array}$ & $\overline{\boldsymbol{E}}_{2 \text { in }}[\%]$ & $\begin{array}{c}\bar{\eta}_{E 2 i n} \\
{[\%]}\end{array}$ & $\begin{array}{l}\overline{\boldsymbol{E}}_{1 \text { in }} \\
{[\%]}\end{array}$ & $\begin{array}{c}\overline{\boldsymbol{\eta}}_{E 1 i n} \\
{[\%]}\end{array}$ \\
\hline \multirow{2}{*}{ MG1: $80 \mathrm{Ar} 20 \mathrm{He}$} & $H I a=2,42 \pm 0,13$ & 168,4 & 94,5 & 267,5 & 80,6 & 9,3 & 55,5 & 15,1 & 94,4 \\
\hline & $H I b=2,19 \pm 0,04$ & 162,4 & 91,1 & 273,4 & 82,4 & 9,9 & 59,3 & 12,7 & 79,6 \\
\hline \multirow{2}{*}{ MG2: $60 \mathrm{Ar} 40 \mathrm{He}$} & $H I a=2,91 \pm 0,02$ & 162,4 & 91,1 & 261,8 & 78,9 & 8,8 & 52,3 & 11,8 & 74,1 \\
\hline & $H I b=2,78 \pm 0,13$ & 162,2 & 91,1 & 219,5 & 66,2 & 5,0 & 30,0 & 7,7 & 48,1 \\
\hline MG3: & $H I a=3,18 \pm 0,07$ & 164,9 & 92,5 & 244,9 & 73,8 & 7,7 & 45,8 & 13,1 & 82,1 \\
\hline $80 \mathrm{Ar} 19 \mathrm{He} \mathrm{O}_{2}$ & $H I b=2,41 \pm 0,15$ & 170,3 & 95,6 & 250,7 & 75,5 & 6,7 & 40,0 & 11,3 & 71,0 \\
\hline MG4: & $H I a=3,14 \pm 0,02$ & 167,3 & 93,9 & 237,7 & 71,6 & 5,4 & 32,3 & 9,7 & 61,1 \\
\hline $60 \mathrm{Ar} 39 \mathrm{He} 1 \mathrm{O}_{2}$ & $H I b=2,80 \pm 0,04$ & 165,2 & 92,7 & 261,0 & 78,7 & 7,4 & 44,4 & 11,5 & 72,2 \\
\hline
\end{tabular}


base. Por último, se determinó la variación de la microdureza Vickers (HV) en las regiones de soldadura. Los resultados relacionados con las discontinuidades de la unión, obtenidos por inspección visual y rayos- $X$, la cualificación es cualitativa en función de los resultados de las muestras analizadas, se muestran en la Tabla 4.

De la Tabla 4 se observa para los aportes térmicos altos que la mayor presencia de poros corresponde a la mezcla MG2. No se observan poros en las mezclas con oxígeno. Para los aportes térmicos bajos, la mayor presencia de poros corresponde a las mezclas sin oxígeno MG1y MG2. En las mezclas con oxígeno se presenta porosidad aislada cuando se usa la mezcla MG3. En la mezcla MG4 se observa la probable presencia de inclusiones solidas de $\mathrm{Al}_{2} \mathrm{O}_{3}$, no identificadas con la técnica de microscopia óptica. De las Tablas 3 y 4, se aprecia como la presencia de $\mathrm{O}_{2}$ permite mayores niveles de energía durante la soldadura, favoreciendo así que haya menor presencia de poros en el metal de soldadura depositado y dando a la vez mayor estabilidad de arco.

Mediante ensayos de tensión transversal se obtuvieron los indicadores de resistencia última, resistencia a fluencia y ductilidad en secciones de deformación de 1,0 y 2,0 pulgadas de la unión soldada (véase la Tabla 5).

En la Tabla 5 se observa uniformidad en los valores de resistencia obtenidos en términos de la resistencia a fluencia $\left(\mathrm{S}_{\mathrm{y}}\right)$, con valor promedio total de $165,4 \pm 3,1 \mathrm{MPa}$ y eficiencia cercana al 93\%. Comparando los valores de resistencia obtenidos con los de la literatura (véase Tabla 1), se observa que estos son significativamente mayores, favoreciendo los indicadores de soldabilidad con proceso GMAW-P. Para las juntas soldadas con aportes térmicos altos el máximo valor de es de 168,4 MPa con una eficiencia del 94,5\% que corresponde con la mezcla MG1:80Ar20He y el más bajo de $162,4 \mathrm{MPa}$ con una eficiencia del 91,1\% para la mezcla MG2:60Ar40He. Para las juntas soldadas con aportes térmicos bajos el máximo valor es de 170,3 MPa con una eficiencia del 95,6\% corresponde con la mezcla MG3:80Ar19 $\mathrm{He}_{1} \mathrm{O}_{2}$ y el más bajo de 162,4 MPa con una eficiencia del $91,1 \%$ para la mezcla MG2:60Ar40He. Al analizar los valores de resistencia obtenidos en términos de la resistencia última $\left(S_{u}\right)$ se observa que para las juntas soldadas con aportes térmicos altos, véase Tabla 5, el máximo valor de $S_{u}$ se de 267,5 MPa con una eficiencia del $80,6 \%$ que corresponde con la mezcla MG1:80Ar20He y el más bajo de 237,7 MPa con una eficiencia del 71,6\% para la mezcla MG4:60Ar39He1O 2 . Para las juntas soldadas con aportes térmicos bajos el máximo valor de $\mathrm{S}_{\mathrm{u}}$ es de 273,5 MPa con una eficiencia del $82,4 \%$ que corresponde con la mezcla MG1:80Ar20He y el más bajo de 219,5 MPa con una eficiencia del 66,6\% para la mezcla MG2:60Ar40He. Se observa uniformidad en los valores de ductilidad (porcentaje de elongación) en secciones de deformación de 1,0 y 2,0 pulgadas, con una reducción alrededor del 55\% y 23\%, respectivamente, con relación a los valores de ductilidad del metal base. Los mayores valores de ductilidad corresponden al uso de la mezcla MG1.

Los valores de microdureza de las regiones de soldadura para las juntas soldadas con aporte térmico alto se muestran en la tabla 6. Del análisis de resultados de los barridos de microdurezas, mostrados en la tabla 6 , se observa uniformidad en los valores de microdureza Vickers obtenidos en cada región de soldadura, con altos valores de eficiencia cercanos al $90 \%$ en promedio para el depósito de soldadura, 92 \% para la región parcialmente fundida - ZPF y al $95 \%$ en la ZAT, mostrando claramente un

Tabla 6. Influencia de la mezcla de gas, con aporte térmico alto, en el valor de la microdureza de las regiones de soldadura de la AA5083-O. ZPF=Zona Parcialmente Fundida, ZAT=Zona Afectada Térmicamente. $\overline{\boldsymbol{M H V}}_{\mathbf{1 0 0} \mathbf{g} / \mathbf{1 0 s}}=$ Microdureza Vickers promedio con carga de $100 \mathrm{~g}$ durante $10 \mathrm{~s}$.

\begin{tabular}{|c|c|c|c|c|c|c|c|c|c|}
\hline \multirow{3}{*}{ Mezcla de gas } & \multicolumn{9}{|c|}{ Región de soldadura - lado de la cara } \\
\hline & \multicolumn{3}{|c|}{ Depósito de Soldadura } & \multicolumn{3}{|c|}{ ZPF } & \multicolumn{3}{|c|}{ ZAT** } \\
\hline & $\begin{array}{l}\text { Extensión* } \\
{[\mathrm{mm}]}\end{array}$ & $\begin{array}{c}\overline{H V} \\
100 \mathrm{~g} / 10 \mathrm{~s}\end{array}$ & $\eta$ & $\begin{array}{c}\text { Extensión } \\
{[\mathrm{mm}]}\end{array}$ & $\begin{array}{c}\overline{H V} \\
100 \mathrm{~g} / 10 \mathrm{~s}\end{array}$ & $\eta$ & $\begin{array}{c}\text { Extensión } \\
{[\mathrm{mm}]}\end{array}$ & $\begin{array}{c}\overline{H V} \\
100 \mathrm{~g} / 10 \mathrm{~s}\end{array}$ & $\eta$ \\
\hline MG1:80Ar20He & 3,7 & $80,3 \pm 3,9$ & 92 & 0,3 & $79,5 \pm 1,5$ & 91 & 4,8 & $84,6 \pm 2,7$ & 97 \\
\hline $\mathrm{MG} 2: 60 \mathrm{Ar} 40 \mathrm{He}$ & 2,85 & $76,1 \pm 2,1$ & 87 & 0,45 & $77,2 \pm 1,9$ & 88 & 5,4 & $79,8 \pm 2,7$ & 91 \\
\hline MG3:80Ar19He1O2 & 4,5 & $78,7 \pm 3,4$ & 90 & 0,3 & $83,5 \pm 2,4$ & 95 & 3,9 & $85,3 \pm 2,6$ & 98 \\
\hline MG4:60Ar39He1O2 & 4,65 & $77,1 \pm 2,5$ & 88 & 0,45 & $81,1 \pm 0,9$ & 93 & 3,5 & $81,6 \pm 2,7$ & 94 \\
\hline \multirow{3}{*}{ Mezcla de gas } & \multicolumn{9}{|c|}{ Región de soldadura - lado de la raíz } \\
\hline & \multicolumn{3}{|c|}{ Depósito de Soldadura } & \multicolumn{3}{|c|}{ ZPF } & \multicolumn{3}{|c|}{ ZAT** } \\
\hline & $\begin{array}{l}\text { Extensión* } \\
{[\mathrm{mm}]}\end{array}$ & $\begin{array}{c}\overline{H V} \\
100 \mathrm{~g} / 10 \mathrm{~s}\end{array}$ & $\eta$ & $\begin{array}{c}\text { Extensión } \\
{[\mathrm{mm}]}\end{array}$ & $\begin{array}{c}\overline{H V} \\
100 \mathrm{~g} / 10 \mathrm{~s}\end{array}$ & $\eta$ & $\begin{array}{c}\text { Extensión } \\
{[\mathrm{mm}]}\end{array}$ & $\begin{array}{c}\overline{H V} \\
100 \mathrm{~g} / 10 \mathrm{~s}\end{array}$ & $\eta$ \\
\hline MG1:80Ar20He & 3,7 & $81,1 \pm 3,4$ & 93 & 0,45 & $83,8 \pm 1,7$ & 96 & 4,5 & $85,6 \pm 2,5$ & 98 \\
\hline MG2:60Ar40He & 3,6 & $75,4 \pm 2,3$ & 86 & 0,45 & $78,6 \pm 3,9$ & 90 & 4,6 & $78,9 \pm 2,1$ & 90 \\
\hline MG3:80Ar19He1O2 & 4,05 & $75,9 \pm 4,1$ & 87 & 0,15 & $77,8 \pm 7,9$ & 89 & 4,5 & $82,8 \pm 4,1$ & 95 \\
\hline MG4:60Ar39He1O2 & 3,35 & $76,8 \pm 1,6$ & 88 & 0,3 & $80,3 \pm 0,9$ & 92 & 5 & $81,3 \pm 3,0$ & 93 \\
\hline
\end{tabular}

*: Medido desde el centro del depósito de soldadura. **: Después de esta zona se localiza el metal base en un límite superior a la extensión de las muestras ( $>15 \mathrm{~mm}$, medido desde el centro del depósito de soldadura) 
ligero ablandamiento de las soldaduras. Del lado de la cara de soldadura: El menor ablandamiento en la región del depósito de soldadura se obtuvo con aporte térmico alto, bajo las mezclas MG1y MG3 con una eficiencia del 92 y $90 \%$ respectivamente. El más alto comportamiento de la microdureza Vickers, en la ZAT se obtuvo en las muestras soldadas con aporte térmico alto, bajo las mezclas MG3:80 $\mathrm{Ar}_{19} \mathrm{He}_{10} \mathrm{O}_{2}$ y MG1:80Ar20 He con valor de 85,3 y $84,6 \mathrm{HV}$ y una eficiencia del 98 y $97 \%$ respectivamente. El valor más bajo de $79,8 \mathrm{HV}$ con una eficiencia del $91 \%$ se obtuvo para la mezcla MG2:60Ar40He con aporte térmico alto.

Del lado de la raíz de soldadura, el menor ablandamiento en la región del depósito de soldadura se obtuvo bajo las mezclas sin oxígeno con una eficiencia cercana al 93\%. Los valores más bajos y próximos entre sí, se obtuvieron bajo las mezclas con presencia de oxígeno. El comportamiento de la microdureza Vickers en la ZAT es muy similar para cada mezcla de gas con una eficiencia cercana al $95 \%$ en promedio.

\subsection{Caracterización microestructural de las uniones soldadas.}

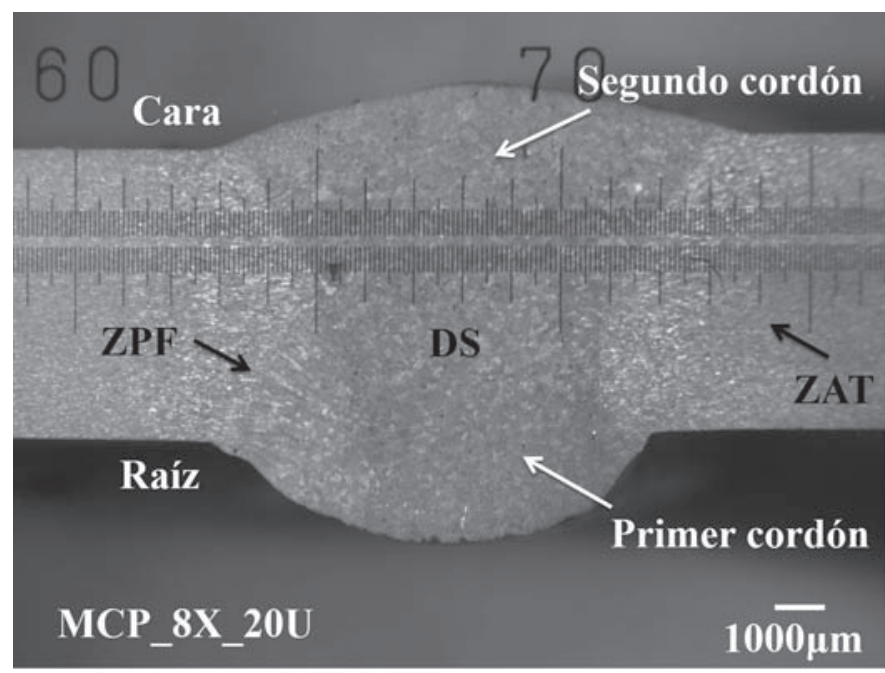

Figura 3. Macroataque de junta soldada GMAW-P automatizado. Ataque Tucker`s. Magnificación $8 X$. DS = Depósito de soldadura, ZPF = Zona parcialmente fundida, ZAT $=$ Zona afectada térmicamente
A partir del análisis macroscópico se observa como el contorno de la cara de raíz de soldadura adoptó la forma del respaldo cerámico utilizado durante la soldadura configurando una apariencia uniforme al cordón, como se ve en la Figura 3. La macrografía de la unión soldada permite la observación de regiones de soldadura, así como la extensión de cada una de estas, siendo además el punto de referencia de pruebas posteriores para el análisis microestructural por microscopia óptica y barridos de microdurezas.

El análisis general de la microestructura ha tomado en cuenta los lados de cara y raíz de la soldadura, donde es posible observar de forma conjunta las regiones de soldadura y características presentes.

Mezclas sin Oxígeno. Para la mezcla MG1: 80Ar20He se observan porosidades esféricas mayormente distribuidas en la región del segundo pase de soldadura, como se observa en la Figura 4a. En la región del pase de raíz, como se muestra en la Figura $4 \mathrm{~b}$, se encuentra poros de menor dimensión con respecto a los del segundo pase de relleno y presentación. Se observan grietas, posiblemente originadas por los altos gradientes de temperatura alcanzados por efectos del segundo pase sobre el primero. Para la mezcla MG2: 60Ar40He se observa la presencia de poros en cantidades menores comparadas con las muestras analizadas de la mezcla MG1, como se muestra en la Figura 4c. Además, se observó del lado de la raíz de soldadura (primer pase) para el aporte térmico la extensión de grietas siguiendo la trayectoria de poros.

Mezclas con Oxígeno. Para la mezcla MG3: $80 \mathrm{Ar} 19 \mathrm{He}_{1} \mathrm{O}_{2}$ se observa en la figura 5a, la presencia de poros en menor cantidad y de menores tamaños en comparación con las mezclas sin oxígeno, véase figura 4 . Además, se observa del lado de la raíz de soldadura (primer pase) para el aporte térmico zonas de apariencia oscura, como se muestra en la Figura 5b, que puede interpretarse como la extensión de grietas siguiendo la trayectoria de poros. Para la mezcla MG4: $60 \mathrm{Ar} 39 \mathrm{He}_{1} \mathrm{O}_{2}$ se observa la probable presencia de inclusiones solidas de $\mathrm{Al}_{2} \mathrm{O}_{3}$, véase Figura $5 \mathrm{c}$, no identificadas con la técnica de microscopia óptica distribuidas en la zona del depósito de soldadura. Lo anterior concuerda con los resultados de la evaluación de discontinuidades y análisis de sanidad de la zona fracturada en los ensayos de tensión (véase tabla 4).

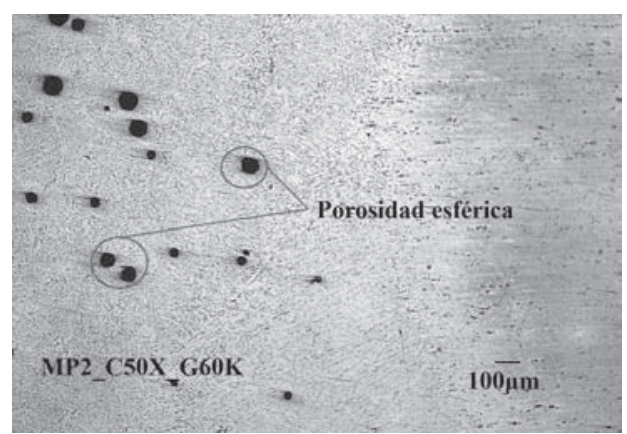

a.

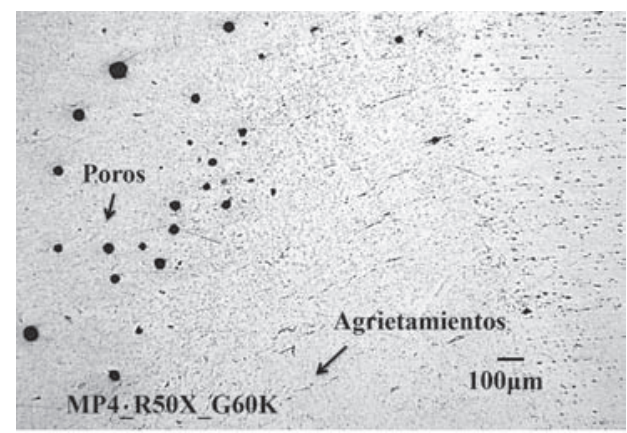

b.

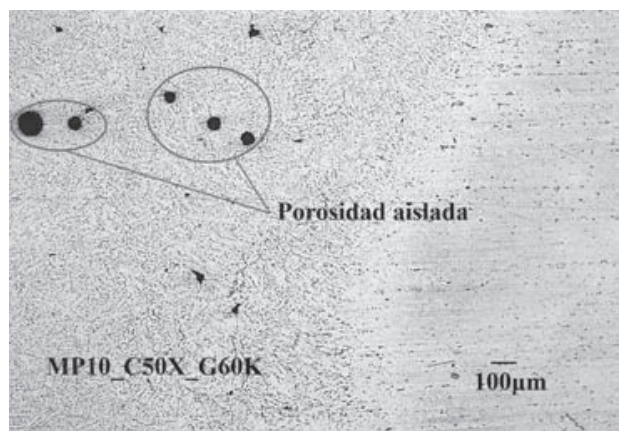

C.

Figura 4. Imagen de microscopia óptica. (a) Segundo pase - MG1:80Ar 20He. (b) Primer pase - MG1:80Ar 20He (c) Segundo pase MG2:60Ar 40He. Ataque Keller's. Magnificación 50X [32] 


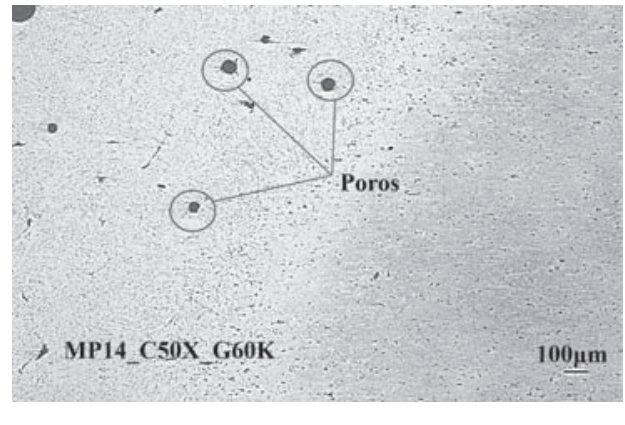

a.

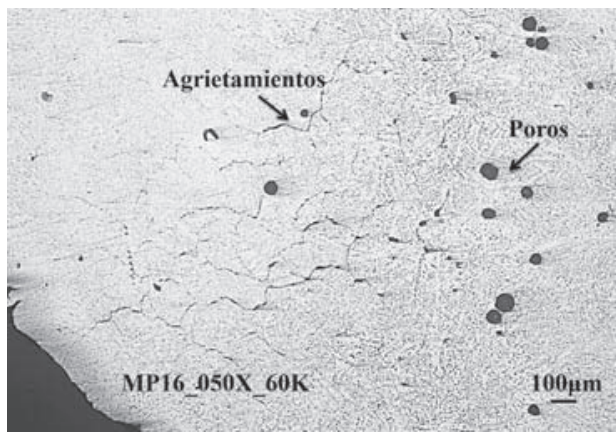

b.

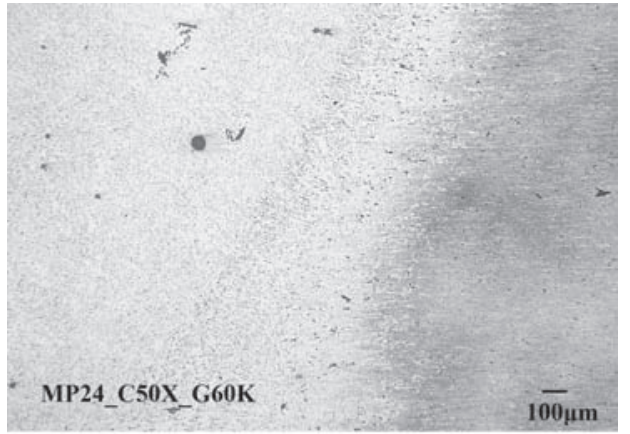

C.

Figura 5. Imagen de de microscopia óptica. (a) Segundo pase - MG3: 80Ar 19He1O $\mathrm{H}_{2}$ (b) Primer pase - MG3: 80Ar 19He1O Segundo pase - MG4: 60Ar 39He1O2. Ataque Keller's. Magnificación 50X [32]

De los resultados obtenidos se observó que las mezclas de Ar-He producen en el depósito de soldadura la mayor presencia de poros en comparación con las mezclas de Ar-He- $\mathrm{O}_{2}$, siendo la MG1:80Ar $20 \mathrm{He}$ la que presenta mayor porcentaje y tamaño de poros en congruencia con lo reportado en la literatura $[2,4]$. Además, con la adición de oxígeno la presencia de poros aparece en niveles cualitativamente más bajos, estos es, son aislados y de menor tamaño. En las soldaduras con $\mathrm{Ar}-\mathrm{He}-\mathrm{O}_{2}$ se observa en la zona del depósito de soldadura, en mayor cantidad bajo la mezcla MG4:60Ar-39He- $1 \mathrm{O}_{2}$, la probable presencia de inclusiones solidas de $\mathrm{Al}_{2} \mathrm{O}_{3}$, resultado que puede ser compatible con lo reportado en la literatura [5,7], La mayor presencia de grietas del lado de la raíz en algunas muestras se puede explicar por desprendimientos localizados e inapropiado sello del respaldo de protección del cordón de raíz y entradas de aire durante la soldadura en estas zonas. Sin embargo, precisar el tipo de discontinuidad, la determinación de causas del agrietamiento y el tipo de partículas presentes sin el acompañamiento de análisis de microscopia electrónica de barrido (SEM) y de composición química por espectrometría dispersiva de Energía (EDS) rebasa el alcance de esta investigación para esta fase experimental de soldaduras.

\subsection{Costo de los gases}

Los gases utilizados fueron suministrados por un proveedor multinacional reconocido que garantiza y certifica la composición y calidad de las mezclas. Para el cálculo se tomó en cuenta: el precio del gas dado por el proveedor para cada mezcla, la velocidad de flujo de gas correspondiente con un valor de $0,01652 \mathrm{~m}^{3} / \mathrm{min}$ y un tiempo de soldadura de un minuto para los cálculos, lo anterior multiplicado por tiempo real de ejecución de las soldaduras. Con respecto al costo de los gases, las mezclas que contienen mayor porcentaje de argón tienen menor valor y entre estas, tienen ligeramente un mayor valor, cercano al 8\%, las mezclas que contienen oxígeno.

\section{Conclusiones}

Se evidenció que las mezclas de Ar-He- $\mathrm{O}_{2}$ originan menor presencia de poros en el depósito de soldadura. Al comparar los valores de resistencia a fluencia de las juntas soldadas en esta investigación con los reportados en la literatura, se observa que los primeros son mayores, favoreciendo los indicadores de soldabilidad con proceso GMAW-P. Al comparar los valores de indicadores de resistencia fluencia y dureza para cada mezcla de gas, estos son muy similares entre sí. Al comparar los valores de ductilidad se encuentra que son mayores en las mezclas con mayor contenido de argón. Por último, se establece acorde con los resultados de esta investigación que el uso de mezclas de Ar-He- $\mathrm{O}_{2}$ favorece la soldabilidad de las juntas soldadas en aluminio naval con proceso GMAW-P automatizado y material de aporte ER5183, encontrándose en la mezcla $80 \mathrm{Ar} 19 \mathrm{He}_{2} \mathrm{O}_{2}$ el gas de protección más adecuado para soldar la aleación de aluminio AA5083.

\section{Agradecimientos}

Los autores agradecen a la Universidad del Norte, a la Universidad Autónoma del Caribe, a la empresa COTECMAR, al SENA- Centro Colombo Alemán y CNPEM/ABTLuS y su Laboratório Nacional de Nanotecnologia - LNNano en Campinas, Brasil, por el apoyo financiero y logístico en el proyecto. Así mismo a las personas José Alfredo Morales, José David Quintero, Milena Ortega y Edinson Samaca de Cotecmar por la logística en esta fase del proyecto. Agradecimiento a los estudiantes de Ingeniería Mecánica de la Universidad Autónoma del Caribe: Halley Vásquez y Julio Loaiza, por su apoyo en la caracterización microestructural.

\section{Referencias Bibliográficas}

[1] MAURY R. H., NIEBLES NUÑEZ E. E. y TORRES SALCEDO J., Diseño para la fabricación y ensamble de productos soldados: Un enfoque metodológico y tecnológico, Barranquilla: Uninorte, 2009.

[2] BLONDEAU R., Ed., Metallurgy and mechanics of welding: processes and industrial applications, London: ISTE-John Wiley \& Sons, 2008.

[3] KUK J., JANG K., LEE D. Y KIM I., Effects of temperature and shielding gas mixture on fatigue life of 5083 aluminum alloy, Journal of Materials Processing Technology, vol. 155- 
156, pp. 1408-1414, 2004.

[4] KAH P.y MARTIKAINEN J., Influence of shielding gases in the welding of metals, International Journal of Advanced Manufacturing Technology, vol. 64, $\mathrm{n}^{\circ}$ 9-12, pp. 1411-1421, 2012.

[5] FORTAIN J.-. M. y LEFEBRE P., Process for the MIG welding of aluminum and its alloys with a shielded gas of the AR/HE/O2 type. United States Patente US 6, 586,700 B2, 2003. [6] ABELLÓ LINDE, Gases de protección para el soldeo MIG de aleaciones no férreas. Gases de protección para la soldadura, Linde, 2004.

[7] LANCASTER J. F., Metallurgy of Welding, Sexta Edicion ed., vol. Sexta Edicion, Abington, Cambridge: Woodhead Publishing Limited, 1999.

[8] BALASUBRAMANIAN V., RAVISANKAR V. y MADHUSUDHAN REDDY G., Effect of pulsed current and post weld aging treatment on tensile properties of argon arc welded high strength aluminium alloy, Materials Science and Engineering: A, vol. 459, $\mathrm{n}^{\mathrm{o}}$ 1-2, pp. 19-34, 2007.

[9] FLETCHER M. J., Glass fibre tape materials - the costeffective solution to weld backing, Welding and Cutting, $\mathrm{n}^{\circ} 5$, pp. 264-265, 2006.

[10] CERAMITEC BACKING, Backing ceramicc for welding. Carbo-Welding Group, Sevilla.

[11] «CAYE. COMERCIAL AUTÓGENA Y ELECTRICA S.A.,» [En línea]. Available: http://www.caye.es/refrac_t_back. pdf. [Último acceso: 3 mayo 2012].

[12] RANDOLPH KISSELL J. y FERRY R. L., Aluminum Structures: A guide to their specifications and design, New York: Jhon Wiley \& Sons, Inc, 2002.

[13] SHIP STRUCTURE COMMITTEE, SSC-452. Aluminum structure design and fabrication guide, Ship Structure Committee, 2007.

[14] THE ALUMINUM ASSOCIATION, Aluminum Design Manual, The Aluminum Association, 2005.

[15] ASM INTERNATIONAL, ASM Handbook. Volume 2: Properties and Selection: Nonferrous Alloys and SpecialPurpose Materials. ASM International, 1992.

[16] ASM INTERNATIONAL, ASM Handbook. Volume 9: Metallography and Microstructures, ASM International, 1992.

[17] PRAVEEN P., YARLAGADDA P. y KANG M., Advancements in pulse gas metal arc welding, Journal of Materials Processing Technology, Vols. \%1 de \%2164-165, p. 1113-1119, 2005.

[18] PALANI P. y MURUGAN N., Selection of parameters of pulsed current gas metal arc welding, Journal of Materials Processing Technology, vol. 172, nº 1, pp. 1-10, 2006.

[19] LINCOLN ELECTRIC, GMAW Welding Guide. Gas Metal Arc Welding Carbon, Low Alloy, and Stainless Steels and Aluminum, J. Nadzam, Ed., LINCOLN ELECTRIC, 2006.

[20] MILLER, Optima-Manual del operador, Soldadura MIG pulsada, Appleton: Miller Electric Mfg. Co., 2002.

[21] CH. GRELLA y MIGUEL A., Selección de los parámetros de pulso en la soldadura de aleaciones al-si y Al-Mg con el proceso GMAW-P, Caracas, 2001.

[22] POSINASETTI PRAVEEN K. M. y PRASAD Y., Behaviour of metal transfer modes in pulse gas metal arc welding of aluminum, de Proceedings of 2006 Advanced Materials Processing Technologies Conference,, Las Vegas, USA, 2006.

[23] DEVAKUMARAN K. y GHOSH P. K., Thermal Characteristics of Weld and HAZ during Pulse Current Gas Metal Arc Weld Bead Deposition on HSLA Steel Plate, Materials and Manufacturing Processes, vol. 25, $\mathrm{n}^{\mathrm{o}}$ 7, pp. 616-630, 2010. [24] GOYAL V. K., GHOSH P. K. y SAINI J. S., Influence of Pulse Parameters on Characteristics of Bead-on-Plate Weld Deposits of Aluminum and Its Alloy in the Pulsed Gas Metal Arc Welding Process, Metallurgical and Materials Transactions A, vol. 39, n 13, pp. 3260-3275, 2008.

[25] GHOSH P. K., DORN L., DEVAKUMARAN K. y HOFMANN F. Pulsed current gas metal arc welding under different shielding and pulse parameters; Part 1: Arc characteristics, ISIJ International, vol. 49, $\mathrm{n}^{\circ}$ 2, pp. 251-260, 2009.

[26] GHOSH P., DORN L., HÜBNER M. y GOYAL V., Arc characteristics and behaviour of metal transfer in pulsed current GMA welding of aluminium alloy, Journal of Materials Processing Technology, vol. 194, nº 1-3, pp. 163-175, 2007. [27] HIRATA Y., Pulsed arc welding, Welding International, vol. 17, no 2, pp. 98-115, 2003.

[28] SCOTTI A., NASCIMENTO A. S., BATISTA M. A. y NASCIMENTO V. C., Comparacâo métodos para cálculo da potência elêtrica através de uma abordagem experimental / matemática, em processos de soldagem a arco, de $8^{\circ}$ Congresso iberoamericano de engenharia mecanica, Cusco, Peru, 2007.

[29] AMERICAN WELDING SOCIETY -AWS, AWS D1.2/ D1.2M:2003 Structural Welding Code - Aluminum, Miami, Florida: American Welding Society, 2003.

[30] ASTM INTERNATIONAL, E384_11. Standard Test Method for Knoop and Vickers Hardness of Materials, ASTM International, 2011.

[31] ASTM INTERNATIONAL, E407-07 Standard practice for microetching metals and alloys, ASTM International, 2007.

[32] LOAIZA RODRÍGUEZ J. A. y VÁSQUEZ SÁNCHEZ H., Caracterización microestructural de la unión soldada en aluminio Al5086-H116 con proceso GMAW-P automatizado, Universidad Autónoma del Caribe, Barranquilla, 2012. 